\title{
Processing speed dysfunction is associated with functional corticostriatal circuit alterations in childhood epilepsy with centrotemporal spikes: a PET and fMRI study
}

\author{
Yuting $\mathrm{Li}^{1,2,3,4}$. Teng Zhang ${ }^{1,2,3,4}$. Jianhua Feng ${ }^{5}$. Shufang Qian ${ }^{1,2,3,4}$. Shuang Wu $\mathbf{W}^{1,2,3,4} \cdot$ Rui Zhou $^{1,2,3,4}$.

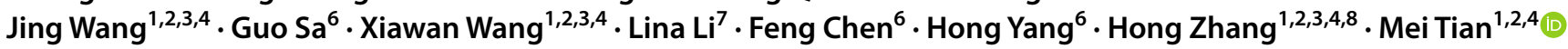

Received: 24 October 2021 / Accepted: 17 February 2022 / Published online: 24 February 2022

(c) The Author(s) 2022

\begin{abstract}
Purpose Epilepsy with centrotemporal spikes (ECTS) is the most common epilepsy syndrome in children and usually presents with cognitive dysfunctions. However, little is known about the processing speed dysfunction and the associated neuroimaging mechanism in ECTS. This study aims to investigate the brain functional abnormality of processing speed dysfunction in ECTS patients by using the ${ }^{18} \mathrm{~F}$-fluorodeoxyglucose $\left({ }^{18} \mathrm{~F}-\mathrm{FDG}\right)$ positron emission tomography (PET) and resting-state functional magnetic resonance imaging (rs-fMRI).

Methods This prospective study recruited twenty-eight ECTS patients who underwent the ${ }^{18} \mathrm{~F}$-FDG PET, rs-fMRI, and neuropsychological examinations. Twenty children with extracranial tumors were included as PET controls, and 20 healthy children were recruited as MRI controls. The PET image analysis investigated glucose metabolism by determining standardized uptake value ratio (SUVR). The MRI image analysis explored abnormal functional connectivity (FC) within the cortical-striatal circuit through network-based statistical (NBS) analysis. Correlation analysis was performed to explore the relationship between SUVR, FC, and processing speed index (PSI).

Results Compared with healthy controls, ECTS patients showed normal intelligence quotient but significantly decreased PSI $(P=0.04)$. PET analysis showed significantly decreased SUVRs within bilateral caudate, putamen, pallidum, left NAc, right rostral middle frontal gyrus, and frontal pole of ECTS patients $(P<0.05)$. Rs-fMRI analysis showed absolute values of 20 FCs were significantly decreased in ECTS patients compared with MRI controls, which connected 16 distinct ROIs. The average SUVR of right caudate and the average of $20 \mathrm{FCs}$ were positively correlated with PSI in ECTS patients $(P=0.034$ and $P=0.005$, respectively).

Conclusion This study indicated that ECTS patients presented significantly reduced PSI, which is closely associated with decreased SUVR and FC of cortical-striatal circuit. Caudate played an important role in processing speed dysfunction.

Clinical trial registration NCT04954729; registered on July 8, 2021, public site, https://clinicaltrials.gov/ct2/show/NCT04 954729
\end{abstract}

Keywords Epilepsy $\cdot$ Positron emission tomography (PET) $\cdot$ Resting-state functional magnetic resonance imaging (Rs-fMRI) · Processing speed

This article is part of the Topical Collection on Neurology

Yuting Li, Teng Zhang and Jianhua Feng contributed equally to this work.

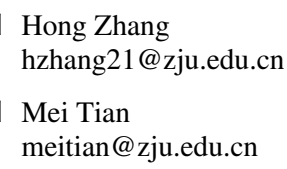

Hong Zhang

hzhang21@zju.edu.cn

Mei Tian

meitian@zju.edu.cn

Extended author information available on the last page of the article

\section{Introduction}

Epilepsy with centrotemporal spikes (ECTS) is the most common focal epilepsy syndrome in children, accounting for $15-20 \%$ of pediatric epilepsy [1]. Although ECTS was originally treated as "benign" due to its relatively low seizure frequency [2], current consensus has reached that ECTS is not benign and can lead to delayed cognitive and behavioral maturation [3, 4]. Language and working memory impairments have been widely investigated in ECTS patients [3, 5]; 
however, little is known about processing speed dysfunction. Processing speed is a key cognitive ability that measures the capacity of decision-making and visual and auditory information processing [6]. Specially, processing speed dysfunction may weaken the learning efficiency of ECTS patients, who are usually school-age children [7]. In order to provide rational bases to improve processing speed, it is necessary to investigate processing speed dysfunction and its associated brain functional abnormality in ECTS patients.

The cortical-striatal circuit provides a physiologic basis for processing speed, as described in previous studies of dementia, trauma, and schizophrenia [8-10]. In this circuit, information is transferred from the cerebral cortex to basal ganglia through the striatum, which can be anatomically divided into caudate, putamen, and nucleus accumbens (NAc), primarily associated with cognitive, motor, and motivational processes, respectively [11]. Particularly, the large amount of interictal epileptic discharges in ECTS patients may disrupt local neuronal activity and functional connectivity of the circuit [12]. Local neuronal activity changes within the circuit can be assessed by glucose metabolic rate using ${ }^{18} \mathrm{~F}$-fluorodeoxyglucose $\left({ }^{18} \mathrm{~F}\right.$-FDG) positron emission tomography (PET) molecular imaging [13]. On the other hand, the functional connectivity alternations can be measured by synchronous fluctuations of blood oxygen level-dependent (BOLD) using resting-state functional magnetic resonance imaging (rs-fMRI) [14]. Furthermore, the combination of PET and rs-fMRI can provide a rich description of functional abnormality for the in-depth investigation of processing speed dysfunction in ECTS patients.

Therefore, in this study, we hypothesize that epileptic discharges of ECTS patients disrupt corticostriatal connections, which is associated with impairments in processing speed. Thus, this study aimed to investigate the glucose metabolism and functional connectivity of cortical-striatal circuit and the relationship with processing speed dysfunction in ECTS patients.

\section{Materials and methods}

\section{Participants}

Thirty patients (13 girls and 17 boys, mean age $=9.8 \pm 2.6$ years) diagnosed as ECTS in our hospital were recruited between June 2019 and March 2021. All patients underwent neuropsychological testing, ${ }^{18} \mathrm{~F}$-FDG PET/CT, rs-fMRI, and structural MRI (sMRI) examinations within 1 month. The inclusion criteria were as follows: (a) clinical diagnosed as ECTS by an experienced pediatric neurologist, including history of tonic-clonic convulsive nocturnal seizures or simple partial seizures during the waking hours and EEG-confirmed classic centrotemporal spikes [15]; (b) age between 6 and 18 years; (c) ${ }^{18}$ F-FDG PET examination more than $48 \mathrm{~h}$ since the last seizure; and (d) no structural abnormalities associated with epilepsy detected on routine MRI. The exclusion criteria included (a) any history of other neurological or psychiatric disorders; (b) pre-scan plasma glucose level $>120 \mathrm{mg} / \mathrm{dl}$ before ${ }^{18} \mathrm{~F}$-FDG PET/CT examination [16]; (c) any contraindications for MRI examination; and (d) head movement (translation $>3 \mathrm{~mm}$ or rotation $>3$ degree) during MRI examination [12]. Two patients were excluded due to movement during resting-state scan. At last, twenty-eight patients were thus included in this study (Fig. 1).

Twenty-three age- and gender- matched healthy children ( 7 girls and 16 boys, mean age $=10.6 \pm 2.2$ years) were enrolled as controls in MRI analysis. The controls underwent neuropsychological testing, $3 \mathrm{~T}$ sMRI, and rs-fMRI examination but except PET examination. Two children were excluded due to movement during resting-state scan, and one child was excluded due to MRI contraindications. To avoid unnecessary radiation, another dataset of 26 age- and gender-matched children with extracranial tumors ( 10 girls and 16 boys, mean age $=10.4 \pm 2.6$ years) was included as controls in PET image analysis [17]. The PET controls had no history of any neurological or psychiatric disorders and never underwent chemotherapy or radiotherapy before PET examination. PET and routine MRI images of these children were carefully reviewed by two experienced physicians to exclude subjects with metabolic or anatomical abnormalities. Discordant results were reviewed by the two physicians to reach a consensus. Six children were excluded due to metabolic abnormalities. In total, twenty healthy children were included in MRI image analysis, and twenty children with extracranial tumors were included in PET image analysis (Fig. 1). The institutional review board approved the present study, and written informed consent was obtained from all participants.

\section{Neuropsychological assessment}

All ECTS patients and MRI controls underwent a comprehensive neuropsychological examination to test cognitive performance. Intelligence was assessed by the Wechsler Children's Intelligence Scale Fourth Edition (WISC-IV), which includes four scales: verbal comprehension index (VCI), perceptual reasoning index (PRI), working memory index (WMI), and processing speed index (PSI) [18].

\section{Image acquisition}

PET images were acquired by a PET/CT scanner (Biograph $\mathrm{mCT}$, Siemens Medical Solutions). All patients fasted for at least $6 \mathrm{~h}$ before injection with a standard dose of ${ }^{18} \mathrm{~F}$ FDG $(3.7 \mathrm{MBq} / \mathrm{kg})$. Then a 5-min brain scan was carried 

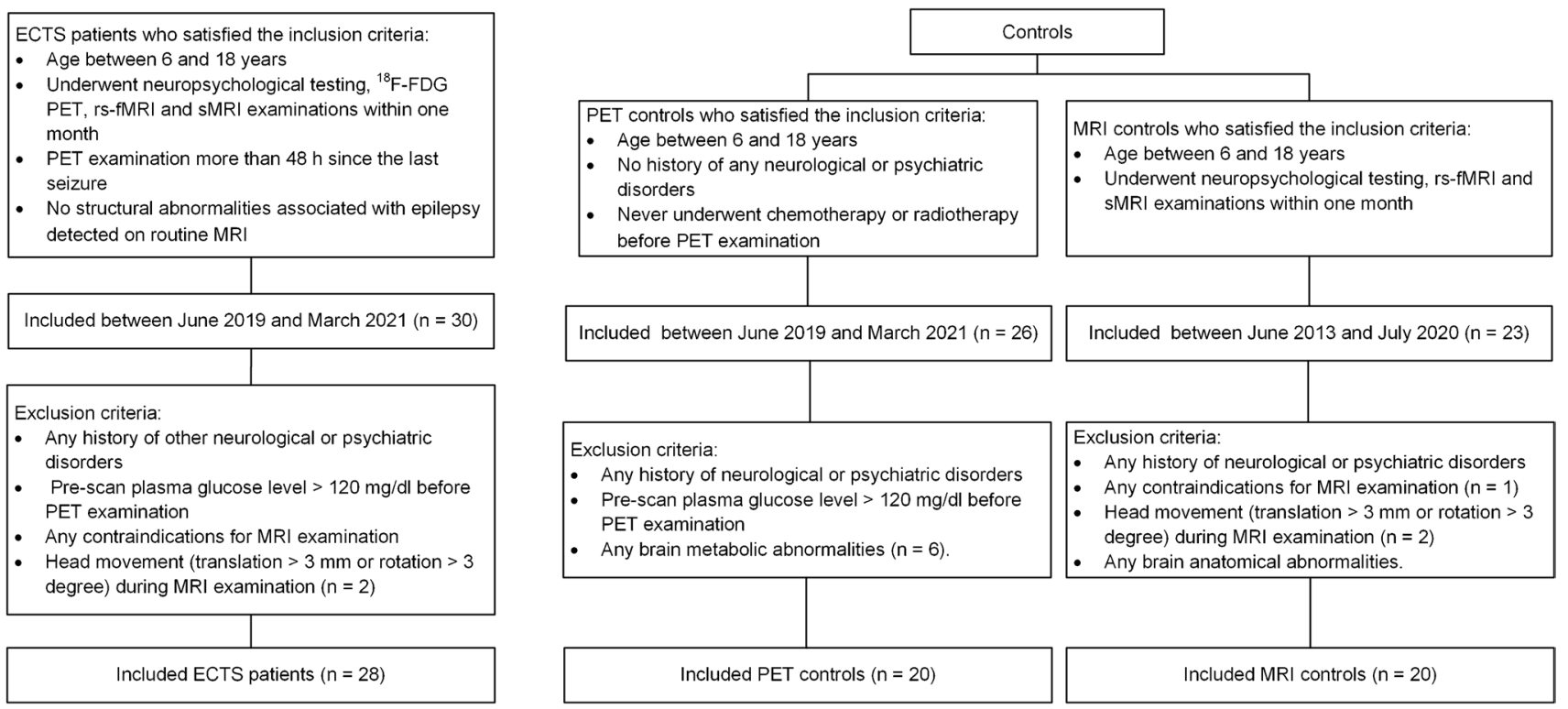

Fig. 1 Flowchart of participants' selection. ECTS, epilepsy with centrotemporal spikes

out approximately 40 min after injection. PET data were obtained after overnight withdrawal of treatment (at least $12 \mathrm{~h}$ ) for ECTS patients.

MRI images were acquired by a $3 \mathrm{~T}$ MRI scanner (GE Medical Systems, Signa HDX, USA) with an 8-channel high-resolution head coil. Earplugs and foam padding were used to limit head movement and reduce scanner noise for the subjects. 3D T1-weighted sMRI images were first acquired using fast spoiled gradient recalled (3D-FSPGR) sequence with the following parameters: 150 sagittal slices (thickness/gap $=1 / 0 \mathrm{~mm})$, repetition time $(\mathrm{TR})=7 \mathrm{~ms}$, echo time $(\mathrm{TE})=2.85 \mathrm{~ms}$, flip angle $=8$ degree, and field of view $($ FOV $)=256 \times 256 \mathrm{~mm}^{2}$. Rs-fMRI scans were acquired using echo-planar images (EPIs) with the following parameters: 29 slices (thickness/gap $=5 / 0 \mathrm{~mm}$ ), TR $=2000 \mathrm{~ms}, \mathrm{TE}=30 \mathrm{~ms}$, $\mathrm{FOV}=240 \times 240 \mathrm{~mm}^{2}$, and flip angle $=90$ degree. During MRI scanning, subjects were instructed to lie still with eyes closed and not to fall asleep.

\section{PET image processing}

${ }^{18}$ F-FDG PET images were preprocessed by Statistical Parametric Mapping (SPM8; https://www.fil.ion.ucl.ac.uk/ spm), FSL (https://fsl.fmrib.ox.ac.uk/fsl/fslwiki) [19], and FreeSurfer (https://surfer.nmr.mgh.harvard.edu/) [20]. All ${ }^{18}$ F-FDG PET images of ECTS patients and controls were spatially normalized to an in-house pediatric ${ }^{18} \mathrm{~F}$-FDG PET template, followed by Gaussian smoothing with 6-mm fullwidth half-maximum (FWHM) [21]. Standardized uptake value ratio (SUVR) was determined to normalize intensities by using cerebellum gray matter as the reference region [22].
In order to validate the hypothesis that the cortical-striatal circuit is the physiologic basis for processing speed in ECTS patients, SPM analysis was first performed to detect all potential metabolic changes across whole brain regions. $P$ value $<0.05$ and cluster size $>100$ were used as thresholds. Then regional analysis was carried out on region of interests (ROIs) which contained at least 100 continuous voxels of SPM clusters, according to the pediatric PET Desikan-Killiany atlas. The ROIs were also thresholded by gray matter (GM) probability $>60 \%$ to reduce the influence of white matter and cerebrospinal fluid retention [23]. The weighted average of regional SUVRs was compared between ECTS patients and controls by two-sample $t$ test, and false positive rate (FDR) was performed to correct multiple comparison. At last, Pearson's correlation between PSI and mean SUVR was investigated for ECTS patients. Construction of pediatric PET template, Desikan-Killiany atlas, and GM probability map was described in the Supplementary Material S1.

\section{MRI processing}

\section{Data preprocessing}

3 T sMRI scans were preprocessed by FreeSurfer (https:// surfer.nmr.mgh.harvard.edu/) [20]. The preprocessing steps included the removal of non-brain tissues, bias field correction, gray-white matter segmentation, gray-white matter boundary tessellation, and tissue segmentation [24]. Whole-brain ROIs were derived in each subject's native space according to the Desikan-Killiany atlas. Rs-fMRI images were preprocessed by DPARSF (https://www.restf 
mri.net) [25] and FSL [19] (https://fsl.fmrib.ox.ac.uk/ fsl/fslwiki). The first 10 images were excluded to reduce magnetization disequilibrium, followed by slice timing correction and head motion correction. The preprocessed sMRI was aligned to corresponding BOLD image by rigid registration using FSL, along with transformation of ROIs according to Desikan-Killiany atlas. BOLD images were then spatial smoothed using a 6-mm FWHM Gaussian kernel, followed by linear detrending and temporal band pass filtering $(0.01-0.08 \mathrm{~Hz})$ to remove low- and high-frequency noises. Nuisance covariates were regressed out to improve the signal-to-noise ratio using the Friston-24 head motion parameters, as well as white matter signal and corticospinal fluid signal. The mean framewise displacements (FDs) of each subject were calculated, and fames with FD $>0.2 \mathrm{~mm}$ were removed to reduce the influence of head movement (scrubbing).

\section{Functional network analysis}

PSI-related functional connectivities (FCs) were compared between ECTS patients and controls by network analysis. For each subject, time series of each node were extracted from preprocessed BOLD images using DPARSF. Pearson correlation was calculated between time series of all ROI pairs according to Desikan-Killiany atlas, and then Fisher's $Z$ transformed as interregional FC. To reduce the influence of spurious interregional connectivity, one-sample $t$ test was performed for each FC in ECTS patients and controls, followed by FDR to correct multiple comparison [26]. Pearson's correlation was estimated between PSI and FCs with $P>0.05$ in either ECTS patients or controls, and then the PSI-related FCs were used in following network-based statistic (NBS) analysis. The NBS analysis involved three major steps [27]. The difference of mean FC between ECTS patients and controls was first determined. Then, nonparametric permutation test was performed by randomly reallocating all subjects into two groups with the same sizes as ECTS and control groups. In each permutation, the mean FC difference was determined to estimate permutation distributions, from which $P$ values were derived according to ranking of differences. At last, multiple comparison was corrected by suprathreshold cluster size test, which extracted the largest connected component from significant connections $(P<0.05)$ in each permutation. The top 5\% largest component size was used as the threshold. Relationships between NBS subnetworks, i.e., the largest connected components, were also investigated.

\section{Statistical analysis}

Statistical analyses of the clinical information were performed using SPSS 22.0 (https://www-01.ibm.com/softw are/analytics/spss). Continuous variables were presented as means \pm standard deviations (SD), and categorical variables were presented as numbers (proportions). Significant differences between patients and controls were compared using two-sample $t$ test for continuous variables and the chi-square test $(\chi 2)$ for categorical variables, respectively. Stepwise multivariate linear regression analysis was performed to evaluate the effects of clinical factors (gender, age at scan, age at seizure onset, seizure duration, the time of AED treatment, antiepileptic drugs, and EEG at diagnosis) on VCI, PRI, WMI, PSI, and full-scale intelligence quotient (FSIQ) in ECTS patients. The influence of seizure duration, time of AED treatment, and AED treatment types (AED-naïve, monotherapy, and polytherapy) on PSI, glucose metabolism, and functional connectivity were also investigated (Supplementary Material S2, S3). Pearson correlation was used in the correlation analysis of ECTS patients. $P<0.05$ was considered statistically significant.

\section{Results}

\section{Clinical information}

The clinical characteristics of ECTS patients and controls were presented in Table 1. The PSI of ECTS patients was significantly decreased than controls $(P=0.04)$, and no significant differences in VCI $(P=0.70)$, PRI $(P=0.32)$, WMI $(P=0.94)$, and FSIQ $(P=0.26)$ were found between ECTS patients and healthy controls. In multivariate linear regression analysis, age at seizure onset was the only significant factor for VCI $(P=0.001)$, PSI $(P=0.015)$, and FSIQ $(P=0.002)$ (Fig. 2).

In patients with ECTS, 39.3\% ( $n=11)$ of patients underwent monotherapy, and $35.7 \%(n=10)$ underwent polytherapy. The most frequently used AED was oxcarbazepine (OXC, $n=13$ ), followed by levetiracetam (LEV, $n=12$ ), valproate (VPA, $n=5$ ), and lamotrigine (LTG, $n=1)$ (Table 2). The seizure duration $(P=0.763)$, time of AED treatment $(P=0.555)$, and AED treatment types showed no significant influence $(P=0.789)$ on PSI.

\section{Metabolic analysis}

Compared with the PET controls, ECTS patients showed distributed hypo-metabolism in the bilateral caudate, putamen, pallidum, lateral orbitofrontal gyri, rostral middle frontal gyri, superior frontal gyri, left thalamus, NAc, right caudal middle frontal gyrus, and right frontal pole (Supplementary Fig. S1). Regional analysis was performed on these ROIs (Table 3), and significantly decreased regional metabolism was found in bilateral caudate, putamen, pallidum, left NAc, right rostral middle frontal gyrus, and frontal 
Table 1 Clinical Information of ECTS patients and controls

\begin{tabular}{llll}
\hline Clinical characteristics & ECTS $(n=28)$ & Controls $(n=20)$ & $P$ value \\
\hline Gender & & 7 & 0.58 \\
Female & 12 & 13 & \\
Male & 16 & $10.75 \pm 2.07$ & 0.27 \\
Age at scan (years) & $9.96 \pm 2.62$ & N/A & - \\
Age at seizure onset (years) & $7.68 \pm 2.92$ & N/A & - \\
Seizure duration (months) & $30.18 \pm 26.26$ & N/A & - \\
The time of AED treatment (months) & $19.39 \pm 26.09$ & & - \\
Antiepileptic drug & & N/A & \\
Monotherapy & 11 & N/A & \\
Polytherapy & 10 & N/A & \\
None & 7 & & \\
Lateralization on EEG at diagnosis & & N/A & \\
Left & 5 & N/A & \\
Right & 8 & N/A & $114.95 \pm 16.38$ \\
Bilateral & 15 & $111.15 \pm 9.83$ & 0.70 \\
Verbal comprehension index & $112.71 \pm 23.95$ & $100.30 \pm 16.13$ & 0.32 \\
Perceptual reasoning index & $107.00 \pm 17.86$ & $102.55 \pm 13.32$ & 0.94 \\
Working memory index & $99.92 \pm 17.82$ & $110.95 \pm 12.10$ & 0.04 \\
Processing speed index & $92.96 \pm 16.88$ & & 0.26 \\
Full-scale intelligence quotient & $105.54 \pm 21.12$ & & \\
\hline
\end{tabular}

ECTS, epilepsy with centrotemporal spikes; $E E G$, electroencephalogram
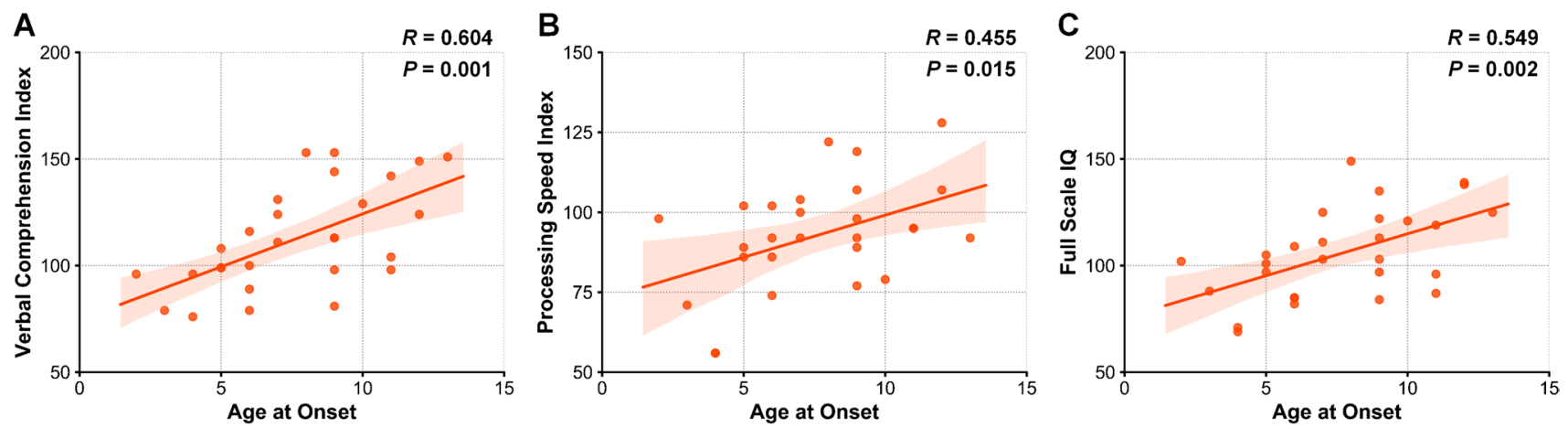

Fig. 2 The relationship between age at onset and IQ of ECTS patients. Age at onset was positively correlated with A VCI; B PSI; and C fullscale IQ. ECTS, epilepsy with centrotemporal spikes; VCI, verbal comprehension index; PSI, processing speed index; IQ, intelligence quotient

Table 2 Information of antiepileptic drugs in patients

\begin{tabular}{ll}
\hline Treatment & $\begin{array}{l}\text { ECTS } \\
(n=28)\end{array}$ \\
\hline AED-naïve & 7 \\
AED treatment & \\
OXC & 5 \\
VPA & 3 \\
LEV & 3 \\
OXC + LEV & 8 \\
VPA + LTG & 1 \\
VPA + LEV & 1 \\
\hline
\end{tabular}

$O X C, \quad$ oxcarbazepine; $V P A$, valproate; $L E V$, levetiracetam; $L T G$, lamotrigine pole of ECTS patients ( $P<0.05$, FDR corrected) (Fig. 3). Pearson's correlation analysis showed that only the SUVR of the right caudate was positively related with PSI $(P=0.034)$ (Fig. 4A). The seizure duration, time of AED treatment, and AED treatment types showed no significant influence on regional SUVR (Supplementary Material S2, S3).

\section{Disrupted network connectivity of ECTS}

The adjacency matrix of ECTS patients and MRI controls were shown in Supplementary Fig. S2. NBS analysis showed that absolute values of 20 FCs were significantly decreased in ECTS patients compared with MRI controls, which connected 
Table 3 Comparison of regional metabolism between ECTS patients and controls

\begin{tabular}{lllll}
\hline ROI & ECTS $(n=28)$ & Controls $(n=20)$ & $\begin{array}{l}P \text { value uncor- } \\
\text { rected }\end{array}$ & $\begin{array}{l}P \text { value } \\
\text { FDR cor- } \\
\text { rected }\end{array}$ \\
\hline Left thalamus & & & 0.321 & 0.341 \\
Left caudate & 1.162 & 1.143 & 0.012 & $0.033^{*}$ \\
Right caudate & 1.170 & 1.226 & 0.011 & $0.033^{*}$ \\
Left putamen & 1.178 & 1.239 & 0.002 & $0.013^{*}$ \\
Right putamen & 1.390 & 1.475 & 0.002 & $0.013^{*}$ \\
Left pallidum & 1.392 & 1.473 & 0.003 & $0.013^{*}$ \\
Right pallidum & 1.128 & 1.197 & 0.002 & $0.013^{*}$ \\
Left accumbens & 1.104 & 1.175 & 0.023 & $0.048^{*}$ \\
Left lateral orbitofrontal & 0.999 & 1.040 & 0.141 & 0.176 \\
Left rostral middle frontal & 1.286 & 1.322 & 0.128 & 0.176 \\
Left superior frontal & 1.499 & 1.546 & 0.191 & 0.216 \\
Right caudal middle frontal & 1.382 & 1.412 & 0.072 & 0.111 \\
Right lateral orbitofrontal & 1.511 & 1.562 & 0.145 & 0.176 \\
Right precentral & 1.255 & 1.287 & 0.352 & 0.352 \\
Right rostral middle frontal & 1.371 & 1.393 & 0.025 & $0.048^{*}$ \\
Right superior frontal & 1.462 & 1.531 & 0.055 & 0.094 \\
Right frontal pole & 1.358 & 1.423 & 0.024 & $0.048^{*}$ \\
\hline
\end{tabular}

$E C T S$, epilepsy with centrotemporal spikes; $F D R$, false discovery rate; $* P<0.05$
16 distinct ROIs (NBS cluster size threshold $=12)($ Table 4$)$. This main subnetwork involved a pathway from rolandic areas to the caudate, thalamus, and the cortical regions, which is part of the cortical-striatal circuit (Fig. 5A). If suprathreshold cluster size test for NBS multiple test correction was avoided, two additional small PSI-related subnetworks could also be found (Fig. 5B-C). The two small subnetworks connected 15 ROIs through 16 FCs (Supplementary Table S1). If PSI-unrelated FCs were also included, connections could be found among the main subnetwork and additional subnetworks (Supplementary Fig. S3). Moreover, ROIs within the main subnetwork was more densely connected than the additional subnetworks.

Absolute values of FCs within the main NBS subnetwork $(P=0.005)$ and the additional subnetwork-2 $(P=0.040)$ were positively correlated with PSI in ECTS patients (Fig. 4B-C). However, the correlation between that within additional subnetwork-1 and PSI was not significant in ECTS patients $(P=0.090)$. In particular, absolute values of FCs between bilateral rolandic areas (precentral and postcentral gyri) were positively correlated with PSI in ECTS patients $(P<0.001)$ (Fig. 4D). The seizure duration, time of AED treatment, and AED treatment types showed no significant influence on PSIrelated FCs (Supplementary Tables S2 and S3).

\section{Discussion}

In this study, we investigated the processing speed dysfunction in ECTS patients using ${ }^{18} \mathrm{~F}$-FDG PET and rsfMRI. Our study showed that the reduced PSI was associated with the decreased SUVR and FC of cortical-striatal circuit in ECTS patients. Particularly, the caudate played an important role in processing speed dysfunction. To the best of our knowledge, this is the first study to investigate functional neuroimaging biomarkers of processing speed dysfunction in ECTS patients.

Most neuroimaging studies concentrated on cognitive impairments of language, attention, and working memory in ECTS patients [1,3]; however, only one study investigated processing speed [21]. Our study, together with the previous study, showed that processing speed could be impaired in patients with ECTS [28]. The functional abnormality of processing speed could be associated with the large amount of epileptic charges during interictal period, despite relatively infrequent seizures in patients with ECTS [12]. In our study, the main PSI-related NBS subnetwork involved FCs between bilateral rolandic areas. 

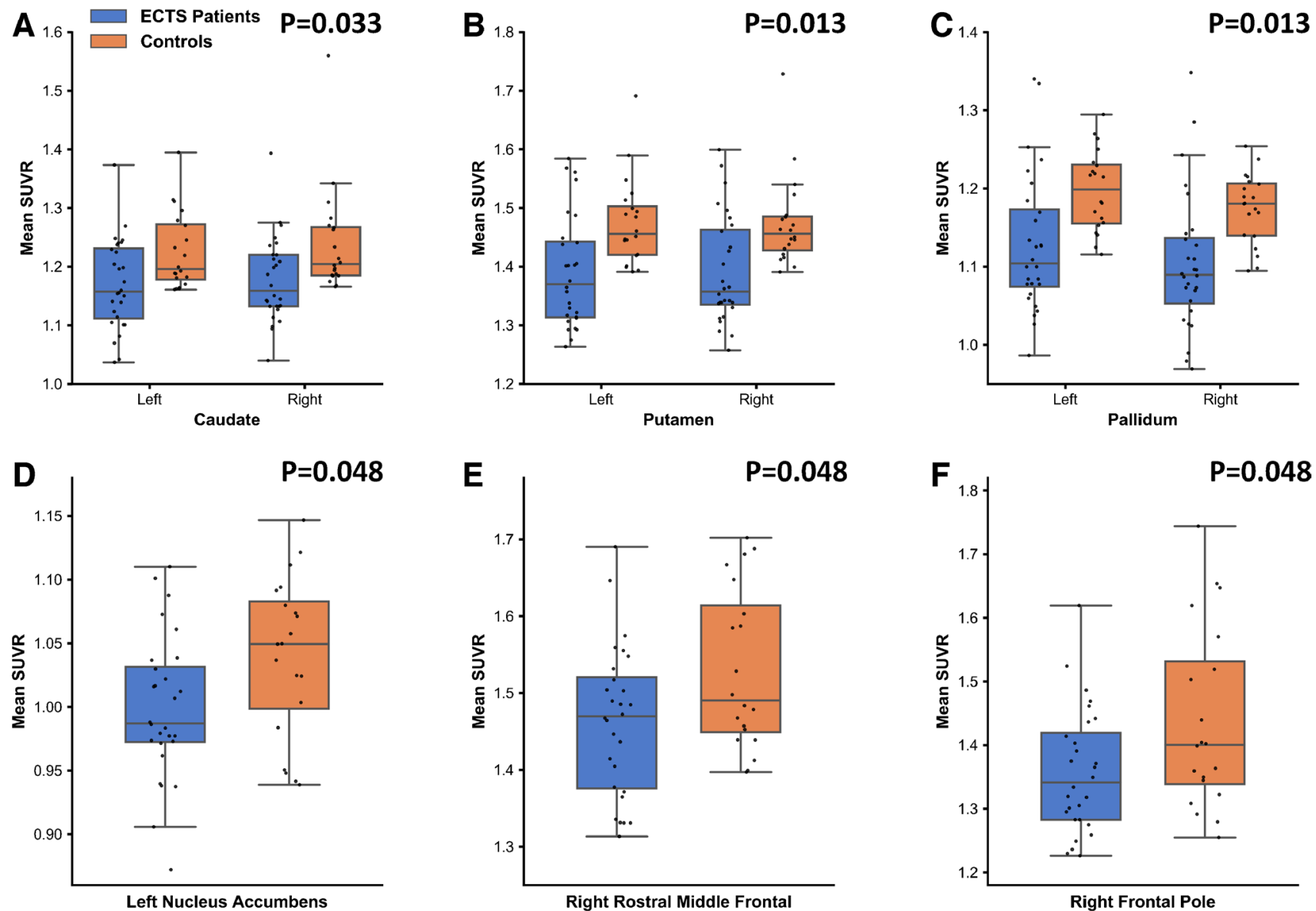

Fig. 3 Significantly decreased SUVRs in ECTS patients compared with controls. A bilateral caudate; B bilateral putamen; C bilateral pallidum; $\mathbf{D}$ left nucleus accumbens; $\mathbf{E}$ right rostral middle frontal; $\mathbf{F}$ right frontal pole
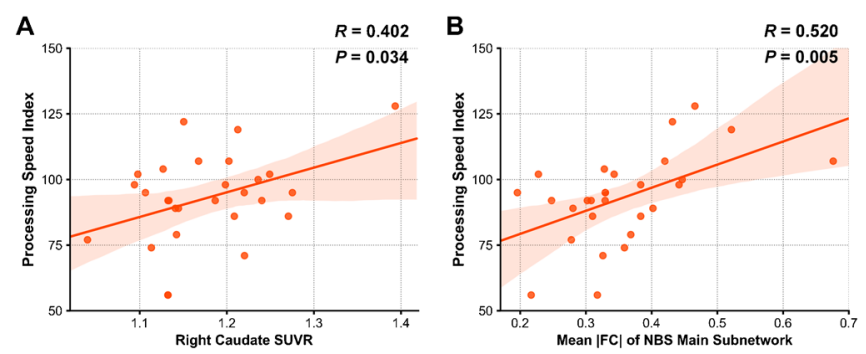

Fig. 4 The relationship between PSI, regional SUVR, and FC in ECTS patients. PSI was positively correlated with A average SUVR of right caudate; $\mathbf{B}$ mean absolute FC within NBS main subnetwork; C mean absolute FC within NBS additional subnetwork-2; and D

Moreover, the decreased absolute FC between bilateral rolandic areas was significantly correlated with reduced PSI in ECTS patients. It could suggest that epileptic discharges originated from rolandic areas result in processing speed dysfunction of ECTS patients.

Our findings indicated that functional abnormality of cortical-striatal circuit was associated with processing
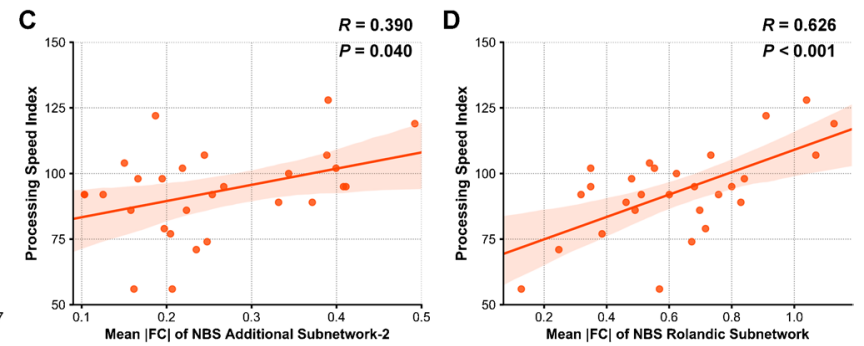

mean absolute FC between bilateral rolandic areas. ECTS, epilepsy with centrotemporal spikes; PSI, processing speed index; SUVR, standardized uptake value ratio; $\mathrm{FC}$, functional connectivity

speed impairment in ECTS patients. The cortical-striatal circuit is the neural pathway connecting cortical regions and basal ganglia to mediate motor, cognitive, and behavioral functions [11]. In this pathway, the bilateral caudate, putamen, pallidum, left NAc, right rostral middle frontal gyrus, and frontal pole showed significantly decreased glucose metabolism in ECTS patients, indicating reduced 
Table 4 Comparison of PSIrelated FC between ECTS patients and controls

\begin{tabular}{|c|c|c|c|c|}
\hline \multicolumn{2}{|l|}{ Functional connectivity } & \multirow[t]{2}{*}{$\operatorname{ECTS}(n=28)$} & \multirow[t]{2}{*}{ Controls $(n=20)$} & \multirow[t]{2}{*}{$P$ value } \\
\hline Region 1 & Region 2 & & & \\
\hline Left thalamus & Left superior temporal & -0.101 & -0.274 & 0.034 \\
\hline Left thalamus & Right middle temporal & -0.116 & -0.292 & 0.024 \\
\hline Left caudate & Left postcentral & -0.185 & -0.409 & 0.004 \\
\hline Right thalamus & Right caudate & 0.487 & 0.844 & 0.006 \\
\hline Right thalamus & Left middle temporal & -0.138 & -0.301 & 0.026 \\
\hline Right thalamus & Left superior temporal & -0.110 & -0.316 & 0.009 \\
\hline Right thalamus & Left frontal pole & -0.021 & -0.198 & 0.007 \\
\hline Right thalamus & Right middle temporal & -0.081 & -0.274 & 0.029 \\
\hline Right thalamus & Right frontal pole & 0.000 & -0.155 & 0.047 \\
\hline Right caudate & Left postcentral & -0.196 & -0.422 & 0.005 \\
\hline Right caudate & Right postcentral & -0.146 & -0.383 & 0.004 \\
\hline Right caudate & Right precentral & -0.141 & -0.310 & 0.029 \\
\hline Left nucleus accumbens & Left middle temporal & 0.049 & -0.123 & 0.009 \\
\hline Left postcentral & Right postcentral & 0.863 & 1.138 & 0.011 \\
\hline Left postcentral & Right precentral & 0.456 & 0.767 & 0.002 \\
\hline Left precentral & Right isthmus cingulate & -0.229 & -0.342 & 0.018 \\
\hline Left precentral & Right postcentral & 0.530 & 0.736 & 0.034 \\
\hline Left precentral & Right precentral & 0.623 & 0.837 & 0.028 \\
\hline Left precuneus & Right isthmus cingulate & 0.509 & 0.725 & 0.019 \\
\hline Right isthmus cingulate & Right precuneus & 0.516 & 0.767 & 0.001 \\
\hline
\end{tabular}

ECTS, epilepsy with centrotemporal spikes; $F C$, functional connectivity; PSI, processing speed index
A

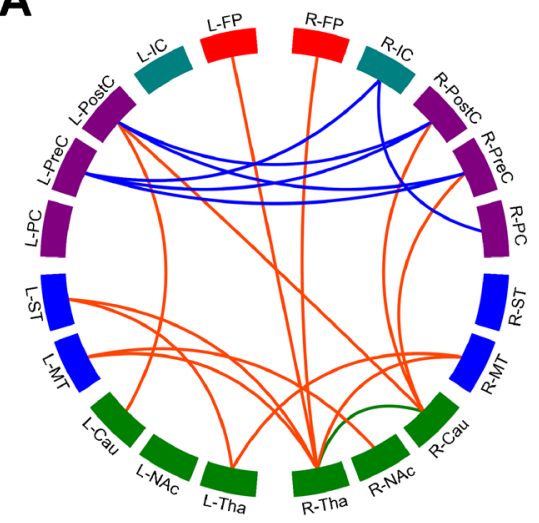

B

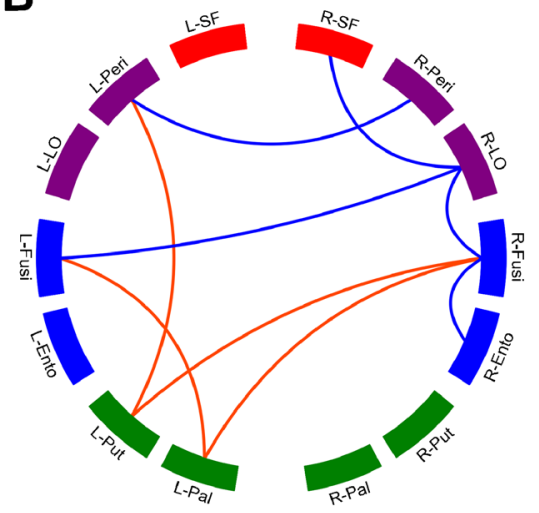

C

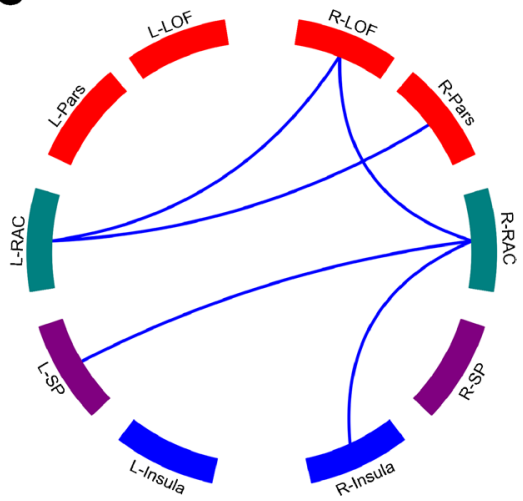

Fig. 5 Network-based statistic analysis results between controls and ECTS patients. Cau, caudate; Ento, entorhinal; FP, frontal pole; Fusi, fusiform; IC, isthmus cingulate; insula, insula; LO, lateral occipital; LOF, lateral orbitofrontal; MT, middle temporal; NAc, nucleus accumbens; Pal, pallidum; Pars, pars triangularis; PC, precuneus; Peri, pericalcarine; PostC, postcentral; PreC, precentral; Put, putamen; RAC, rostral anterior cingulate; SF, superior frontal; SP, superior parietal; ST, superior temporal; Tha, thalamus neuronal activity in these regions [13]. In addition, SUVR in the caudate was positively correlated with PSI. Moreover, the PSI-related NBS main subnetwork involved FCs between the bilateral rolandic areas and FCs from the rolandic areas to the caudate, the thalamus, and then the frontal and temporal lobes. It may indicate how the epileptic discharges influence the cortical-striatal circuit. The caudate could be directly influenced by the epileptic discharges originated from the rolandic areas. This finding is consistent with a previous finding that caudate is particularly vulnerable to pathological factors due to its topological centrality [29]. The decreased SUVR and FC of caudate could therefore suggest that the caudate is a primary structure of processing speed dysfunction in ECTS patients, in consistence with a previous study of processing speed in traumatic brain injury [30]. 
The cortical-striatal circuit from NBS analysis also involved cortical regions including the frontal pole (FP) and the superior temporal (ST) and middle temporal (MT) gyri. The FP played an import role in decision processing [31], and ST and MT gyri were considered to be associated with speech processing [32]. The main NBS subnetwork also involved precuneus and isthmus cingulate, impairments of which were related to slowed processing speed [31, 33]. Moreover, there were significantly decreased connections between NBS main and additional subnetworks, which were not correlated with PSI and thus removed from NBS analysis. Therefore, the two NBS additional subnetworks may be branches of the cortical-striatal circuit in the main subnetwork. The cortical regions in the two branches, including the fusiform, pericalcarine, lateral orbitofrontal, rostral anterior cingulate, and superior parietal, were also considered to be associated with processing speed [33-37].

In this study, ECTS patients were treated with the secondgeneration AEDs, including OXC, LEV, VPA, and LTG. These new AEDs had very little side effects on cognition, and most of them could even protect processing speed ability [38-43]. The group comparison showed no significant differences in PSI among AED-naïve patients and patients who underwent monotherapy and polytherapy. Therefore, the processing speed impairment could be resulted from epileptic discharges rather than AED treatment. The processing speed depends on various functional abilities, such as attention, planning, and visuospatial and auditory skills [6]. These functions are supported by complex brain network that provides the physiological basis for information processing [44]. In this study, the decreased FCs were connecting to regions related to processing speed and formed a subnetwork associated with decreased PSI. It indicated that network deficit may be the pathological basis of processing speed dysfunction in ECTS, similar to other neuropsychological disorders [30, 45]. As pediatric brains are highly plastic, processing speed can be improved like other cognitive abilities. Our findings could provide rational bases to improve processing speed of ECTS patients, such as physical exercise [46], brain training game [47], and transcranial stimulation [48].

On the other hand, AED could still influence glucose metabolism and functional connectivities. VPA could reduce whole-brain glucose metabolism; however, this reduction is very slight at a negligible level [40]. Patients taking LEV showed increased glucose metabolism in the bilateral caudate, frontal lobes, and left parietal lobe [40], and those taking LTG showed reduced glucose metabolism in the thalamus and basal ganglia [49]. In a previous fMRI study, ECTS patients who underwent AED treatment showed decreased FC in default network, while those who did not take AEDs showed increased FC in default network and motion-related networks [16]. Similarly, a combined EEG-fMRI study showed increased whole-brain FC after withdrawal of AEDs in focal epilepsy [50]. Although group comparison showed no significant difference in regional SUVR and FC among AED-naïve patients and patients who underwent monotherapy and polytherapy, the potential influences cannot be totally avoided and formed a major limitation of our study. Similar to previous studies, the AED treatment was specialized by an experienced physician according to patient seizure condition in this study $[17,51]$. Future works may consider to recruit more AED-naïve patients to validate our findings.

\section{Conclusion}

This study indicated that ECTS patients presented significantly reduced PSI, which is closely associated with decreased SUVR and FC of the cortical-striatal circuit. Caudate played an important role in processing speed dysfunction.

Supplementary Information The online version contains supplementary material available at https://doi.org/10.1007/s00259-022-05740-w.

Funding This work is partly sponsored by grants from the National Natural Science Foundation of China (NSFC) (No. 81761148029, $81725009,82030049)$ and the Ministry of Science and Technology (MOST) (No. 2021YFE0108300).

\section{Declarations}

Ethics approval All procedures were performed in accordance with the principles of the 1964 Declaration of Helsinki and its later amendments. The study design was approved by the Institutional Review Board of the Second Hospital of Zhejiang University School of Medicine (Approval No. 2019-266).

Consent to participate For this study, written informed consent was obtained from all participants.

Conflict of interest The authors declare no competing interests.

Open Access This article is licensed under a Creative Commons Attribution 4.0 International License, which permits use, sharing, adaptation, distribution and reproduction in any medium or format, as long as you give appropriate credit to the original author(s) and the source, provide a link to the Creative Commons licence, and indicate if changes were made. The images or other third party material in this article are included in the article's Creative Commons licence, unless indicated otherwise in a credit line to the material. If material is not included in the article's Creative Commons licence and your intended use is not permitted by statutory regulation or exceeds the permitted use, you will need to obtain permission directly from the copyright holder. To view a copy of this licence, visit http://creativecommons.org/licenses/by/4.0/. 


\section{References}

1. Wickens S, Bowden SC, Souza WD. Cognitive functioning in children with self-limited epilepsy with centrotemporal spikes: a systematic review and meta-analysis. Epilepsia. 2017;1-13. https://doi.org/10.1111/epi.13865.

2. Panayiotopoulos CP, Michael M, Sanders S, Valeta T, Koutroumanidis M. Benign childhood focal epilepsies: assessment of established and newly recognized syndromes. Brain. 2008;131:2264 86. https://doi.org/10.1093/brain/awn162.

3. Nickels KC, Zaccariello MJ, Hamiwka LD, Wirrell EC. Cognitive and neurodevelopmental comorbidities in paediatric epilepsy. Nat Rev Neurol. 2016;12:465-76. https://doi.org/10.1038/nrneurol. 2016.98.

4. Li Y, Feng J, Zhang T, Shi K, Ding Y, Zhang X, et al. Brain metabolic characteristics distinguishing typical and atypical benign epilepsy with centro-temporal spikes. Eur Radiol. 2021;31:9335-45. https://doi.org/10.1007/s00330-021-08051-0.

5. Kárpáti J, Donauer N, Somogyi E, Kónya A. Working memory integration processes in benign childhood epilepsy with centrotemporal spikes. Cogn Behav Neurol. 2015;28:207-14. https://doi.org/10.1097/WNN.0000000000000075.

6. Takeuchi H, Kawashima R. Effects of processing speed training on cognitive functions and neural systems. Rev Neurosci. 2012;23:289-301. https://doi.org/10.1515/revneuro-2012-0035.

7. Völkl-Kernstock S, Bauch-Prater S, Ponocny-Seliger E, Feucht M. Speech and school performance in children with benign partial epilepsy with centro-temporal spikes (BCECTS). Seizure. 2009;18:320-6. https://doi.org/10.1016/j.seizure.2008.11.011.

8. Botzung A, Philippi N, Noblet V, De SPL, Blanc F. Pay attention to the basal ganglia : a volumetric study in early dementia with Lewy bodies. Alzheimers Res Ther. 2019;11:108-17. https://doi.org/10.1186/s13195-019-0568-y.

9. Bernick C, Banks SJ, Shin W, Obuchowski N, Butler S, Noback $\mathrm{M}$, et al. Repeated head trauma is associated with smaller thalamic volumes and slower processing speed : the Professional Fighters' Brain Health Study. Br J Sport Med. 2015;49:100711. https://doi.org/10.1136/bjsports-2014-093877.

10. Qi S, Bustillo J, Turner JA, Jiang R, Zhi D, Fu Z, et al. The relevance of transdiagnostic shared networks to the severity of symptoms and cognitive deficits in schizophrenia: a multimodal brain imaging fusion study. Transl Psychiatry. 2020;10:149-59. https://doi.org/10.1038/s41398-020-0834-6.

11. Haber SN. The primate basal ganglia: parallel and integrative networks. J Chem Neuroanat. 2003;26:317-30. https://doi.org/ 10.1016/j.jchemneu.2003.10.003.

12. Ji GJ, Yu Y, Miao HH, Wang ZJ, Tang YL, Liao W. Decreased network efficiency in benign epilepsy with centrotemporal spikes. Radiology. 2017;283:186-94. https://doi.org/10.1148/ radiol.2016160422

13. Verger A, Guedj E. The renaissance of functional 18F-FDG PET brain activation imaging. Eur J Nucl Med Mol Imaging. 2018;45:2338-41. https://doi.org/10.1007/s00259-018-4165-2.

14. Harrison TM, Maass A, Adams JN, Jagust WJ, Du R, Baker SL. Tau deposition is associated with functional isolation of the hippocampus in aging. Nat Commun. 2019;10:4900-12. https:// doi.org/10.1038/s41467-019-12921-z.

15. Engel J. A proposed diagnostic scheme for people with epileptic seizures and with epilepsy: report of the ILAE task force on classification and terminology. Epilepsia. 2001;42:796-803. https://doi.org/10.1046/j.1528-1157.2001.10401.x.

16. Varrone A, Asenbaum S, Vander Borght T, Booij J, Nobili $\mathrm{F}$, Någren $\mathrm{K}$, et al. EANM procedure guidelines for PET brain imaging using [18F]FDG, version 2. Eur J Nucl Med
Mol Imaging. 2009;36:2103-10. https://doi.org/10.1007/ s00259-009-1264-0.

17. Zhu Y, Feng J, Wu S, Hou H, Ji J, Zhang K, et al. Glucose metabolic profile by visual assessment combined with statistical parametric mapping analysis in pediatric patients with epilepsy. $\mathbf{J}$ Nucl Med. 2017;58:1293-9. https://doi.org/10.2967/jnumed.116. 187492.

18. Gomez R, Vance A, Watson SD. Structure of the Wechsler intelligence scale for children - fourth edition in a group of children with ADHD. Front Psychol. 2016; 7:1-11. https://doi.org/10. 3389/fpsyg.2016.00737.

19. Jenkinson M, Beckmann CF, Behrens TEJ, Woolrich MW, Smith SM. FSL Neuroimage. 2012;62:782-90. https://doi.org/10.1016/j. neuroimage.2011.09.015.

20. Fischl B. FreeSurfer. Neuroimage. 2012;62:774-81. https://doi. org/10.1016/j.neuroimage.2012.01.021.

21. Zhang T, Li Y, Zhao S, Xu Y, Zhang X, Wu S, et al. High-resolution pediatric age-specific 18F-FDG PET template: a pilot study in epileptogenic focus localization. Eur J Nucl Med Mol Imaging. 2021. https://doi.org/10.1007/s00259-021-05611-w.

22. BoscoloGalazzo I, Mattoli MV, Pizzini FB, De Vita E, Barnes A, Duncan JS, et al. Cerebral metabolism and perfusion in MRnegative individuals with refractory focal epilepsy assessed by simultaneous acquisition of 18F-FDG PET and arterial spin labeling. NeuroImage Clin. 2016;11:648-57. https://doi.org/10.1016/j. nicl.2016.04.005.

23. Nordberg A, Carter SF, Rinne J, Drzezga A, Brooks DJ, Vandenberghe R, et al. A European multicentre PET study of fibrillar amyloid in Alzheimer's disease. Eur J Nucl Med Mol Imaging. 2013;40:104-14. https://doi.org/10.1007/s00259-012-2237-2.

24. Fischl B, Sereno MI, Dale AM. Cortical surface-based analysis. II: Inflation, flattening, and a surface-based coordinate system. Neuroimage. 1999;9:195-207. https://doi.org/10.1006/nimg.1998. 0396.

25. Yan CG, Di WX, Zuo XN, Zang YF. DPABI: data processing $\&$ analysis for (resting-state) brain imaging. Neuroinformatics. 2016;14:339-51. https://doi.org/10.1007/s12021-016-9299-4.

26. Yang X, Liu J, Meng Y, Xia M, Cui Z, Wu X, et al. Network analysis reveals disrupted functional brain circuitry in drug-naive social anxiety disorder. Neuroimage. 2019;190:213-23. https:// doi.org/10.1016/j.neuroimage.2017.12.011.

27. Zalesky A, Fornito A, Bullmore ET. Network-based statistic: identifying differences in brain networks. Neuroimage. 2010;53:1197207. https://doi.org/10.1016/j.neuroimage.2010.06.041.

28. Fujiwara H, Tenney J, Kadis DS, Byars A, Altaye M, Spencer $\mathrm{C}$, et al. Cortical morphology, epileptiform discharges, and neuropsychological performance in BECTS. Acta Neurol Scand. 2018;138:432-40. https://doi.org/10.1111/ane.12997.

29. Crossley NA, Mechelli A, Scott J, Carletti F, Fox PT, Mcguire $\mathrm{P}$, et al. The hubs of the human connectome are generally implicated in the anatomy of brain disorders. Brain. 2014;137:2382-95. https://doi.org/10.1093/brain/awu132.

30. De Simoni S, Jenkins PO, Bourke NJ, Fleminger JJ, Hellyer PJ, Jolly AE, et al. Altered caudate connectivity is associated with executive dysfunction after traumatic brain injury. Brain. 2018;141:148-64. https://doi.org/10.1093/brain/awx309.

31. White CN, Congdon E, Mumford JA, Karlsgodt KH, Sabb FW, Freimer NB, et al. Decomposing decision components in the Stopsignal task: a model-based approach to individual differences in inhibitory control. J Cogn Neurosci. 2014;26:1601-14. https://doi. org/10.1162/jocn.

32. Cohen L, Dehaene S, Naccache L, Lehéricy S, Dehaene-Lambertz G, Hénaff MA, et al. The visual word form area. Spatial and temporal characterization of an initial stage of reading in normal subjects and posterior split-brain patients. Brain. 2000;123:291-307. https://doi.org/10.1093/brain/123.2.291. 
33. Garcia-Ramos C, Dabbs K, Meyerand E, Prabhakaran V, Hsu D, Jones $\mathrm{J}$, et al. Psychomotor slowing is associated with anomalies in baseline and prospective large scale neural networks in youth with epilepsy. NeuroImage Clin. 2018;19:222-31. https://doi.org/ 10.1016/j.nicl.2018.04.020.

34. Nogueira R, Abolafia JM, Drugowitsch J, Balaguer-Ballester E, Sanchez-Vives MV, Moreno-Bote R. Lateral orbitofrontal cortex anticipates choices and integrates prior with current information. Nat Commun. 2017;8. https://doi.org/10.1038/ncomms14823.

35. Bahlmann J, Blumenfeld RS, D'Esposito M. The rostro-caudal axis of frontal cortex is sensitive to the domain of stimulus information. Cereb Cortex. 2015;25:1815-26. https://doi.org/10.1093/ cercor/bht419.

36. Rosenbaum D, Blum L, Schweizer P, Fallgatter AJ, Herrmann MJ, Ehlis A-C, et al. Comparison of speed versus complexity effects on the hemodynamic response of the trail making test in block designs. Neurophotonics. 2018;5:1. https://doi.org/10.1117/1. nph.5.4.045007.

37. Colom R, Burgaleta M, Román FJ, Karama S, Álvarez-Linera $\mathrm{J}$, Abad FJ, et al. Neuroanatomic overlap between intelligence and cognitive factors: morphometry methods provide support for the key role of the frontal lobes. Neuroimage. 2013;72:143-52. https://doi.org/10.1016/j.neuroimage.2013.01.032.

38. Donati F, Gobbi G, Campistol J, Rapatz G, Daehler M, Sturm Y, et al. Effects of oxcarbazepine on cognitive function in children and adolescents with partial seizures. Neurology. 2006;67:679-82. https://doi.org/10.1212/01.wnl.0000230138.46508.5b.

39. Ciesielski AS, Samson S, Steinhoff BJ. Neuropsychological and psychiatric impact of add-on titration of pregabalin versus levetiracetam: a comparative short-term study. Epilepsy Behav. 2006;9:424-31. https://doi.org/10.1016/j.yebeh.2006.07.011.

40. Leiderman DB, Balish M, Bromfield EB, Theodore WH. Effect of valproate on human cerebral glucose metabolism. Epilepsia. 1991;32:417-22. https://doi.org/10.1111/j.1528-1157.1991.tb046 71.x.

41. Theodore WH, Bromfield E, Onorati L. The effect of carbamazepine on cerebral glucose metabolism. Ann Neurol. 1989;25:51620. https://doi.org/10.1002/ana.410250519.

42. Suo GH, Zheng YQ, Wu YJ, Tang JH. Effects of levetiracetam and oxcarbazepine monotherapy on intellectual and cognitive development in children with benign epilepsy with centrotemporal spikes. Acta Neurol Belg. 2021;121:1265-73. https://doi.org/10.1007/ s13760-021-01613-5.
43. Meador KJ, Loring DW, Hulihan JF, Kamin M, Karim R. Differential cognitive and behavioral effects of topiramate and valproate. Neurology. 2001;60:1483-8. https://doi.org/10.1212/01. wnl.0000063308.22506.19.

44. Bassett DS, Sporns O. Network neuroscience. Nat Neurosci. 2017;20:353-64. https://doi.org/10.1038/nn.4502.

45. Bergsland N, Zivadinov R, Dwyer MG, Weinstock-Guttman B, Benedict RHB. Localized atrophy of the thalamus and slowed cognitive processing speed in MS patients. Mult Scler. 2016;22:1327-36. https://doi.org/10.1177/1352458515616204.

46. Sahnoune I, Inoue T, Kesler SR, Rodgers SP, Sabek OM, Pedersen SE, et al. Exercise ameliorates neurocognitive impairments in a translational model of pediatric radiotherapy. Neuro Oncol. 2018;20:695-704. https://doi.org/10.1093/neuonc/nox 197.

47. Nouchi R, Kawata NYDS, Saito T, Himmelmeier RM, Nakamura $\mathrm{R}$, Nouchi H, et al. Dorsolateral prefrontal cortex activity during a brain training game predicts cognitive improvements after four weeks' brain training game intervention: evidence from a randomized controlled trial. Brain Sci. 2020;10:1-15. https://doi.org/ 10.3390/brainsci10080560.

48. Hausman HK, O'Shea A, Kraft JN, Boutzoukas EM, Evangelista ND, Van Etten EJ, et al. The role of resting-state network functional connectivity in cognitive aging. Front Aging Neurosci. 2020;12:1-10. https://doi.org/10.3389/fnagi.2020.00177.

49. Eun YJ, Woo ST, Seung BH. Regional effects of lamotrigine on cerebral glucose metabolism in idiopathic generalized epilepsy. Arch Neurol. 2006;63:1282-6. https://doi.org/10.1001/archneur. 63.9.1282.

50. Hermans K, Ossenblok P, Van Houdt P, Geerts L, Verdaasdonk $\mathrm{R}$, Boon P, et al. Network analysis of EEG related functional MRI changes due to medication withdrawal in focal epilepsy. NeuroImage Clin. 2015;8:560-71. https://doi.org/10.1016/j.nicl.2015.06. 002.

51. Haneef Z, Lenartowicz A, Yeh HJ, Levin HS, Engel J, Stern JM. Functional connectivity of hippocampal networks in temporal lobe epilepsy. Epilepsia. 2014;55:137-45. https://doi.org/10.1111/epi. 12476.

Publisher's Note Springer Nature remains neutral with regard to jurisdictional claims in published maps and institutional affiliations.

\section{Authors and Affiliations}

\section{Yuting $\mathrm{Li}^{1,2,3,4}$. Teng Zhang ${ }^{1,2,3,4}$. Jianhua Feng ${ }^{5}$. Shufang Qian ${ }^{1,2,3,4}$. Shuang $\mathrm{Wu}^{1,2,3,4} \cdot$ Rui Zhou $^{1,2,3,4}$.

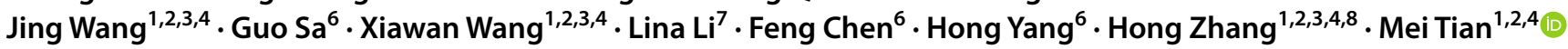

1 Department of Nuclear Medicine and Medical PET Center, The Second Hospital of Zhejiang University School of Medicine, 88 Jiefang Road, Hangzhou 310009, Zhejiang, China

2 Institute of Nuclear Medicine and Molecular Imaging of Zhejiang University, Hangzhou, China

3 Key Laboratory for Biomedical Engineering of Ministry of Education, Zhejiang University, Hangzhou, China

4 Key Laboratory of Medical Molecular Imaging of Zhejiang Province, Hangzhou, China
5 Department of Pediatrics, The Second Hospital of Zhejiang University School of Medicine, Hangzhou, China

6 Department of Radiology, The First Hospital of Zhejiang University School of Medicine, Hangzhou, China

7 College of Medical Imaging, Shanxi Medical University, Taiyuan, China

8 The College of Biomedical Engineering and Instrument Science, Zhejiang University, Hangzhou, China 